\title{
Microcomputed Tomography Technique for In Vivo Three-Dimensional Fat Tissue Volume Evaluation After Polymer Injection
}

\author{
Sarah Berndt, $\mathrm{PhD}$, Ioana Konz, $\mathrm{PhD}$, Didier Colin, $\mathrm{PhD}$, Stéphane Germain, Brigitte Pittet-Cuénod, MD, \\ Harm-Anton Klok, PhD, and Ali Modarressi, MD, PD ${ }^{1}$
}

Tissue engineering technologies are new and promising techniques in fat tissue reconstruction. However, to assess their efficacy before any clinical application, in vivo experiments are mandatory. This study assesses whether microcomputed tomography (CT) scan imaging is suitable to analyze in vivo the behavior of injected engineered polymer and changes in fat tissue. The volume of mice inguinal fat pads and the resorption rate of different polymers were analyzed by CT scan for up to 3 months. Different biomaterials were used, including our innovative microspheres loaded with oleic acid. We were able to follow in vivo the polymer and the fat volume of the same animals during a long-term follow-up of 90 days. Semiautomatic three-dimensional quantification allowed to determine the fat volume enhancement after injection, as well as the resorption rate of our product compared to other biomaterials (i.e., polylactic and hyaluronic acid) until 90 days. Our results demonstrate the encouraging proof-of-principle evidence for the application of micro-CT scan technology to follow in vivo biodegradable polymers in a fat tissue engineering approach. This noninvasive technique offers the advantages of the long-term follow-up of fat tissue and synthetic materials in the same animals, which allows both a scientific evaluation of the measurements and the reduction of the number of animals used in in vivo protocols in accordance with the 3 " $\mathrm{R}$ " principles governing the use of animals in science.

Keywords: noninvasive in vivo imaging, micro-CT scan, PLGA microspheres, adipose tissue engineering

\section{Introduction}

Ooft Tissue ReCONSTRUCtion following trauma or cancer $\checkmark$ treatment or for aesthetic indications is challenging. Autologous fat grafting is now considered as the gold standard technique to augment or fill soft-tissue defects. ${ }^{1}$ However, this surgical approach may not be feasible in the absence of donor sites in thin patients. Moreover, as part of the grafted fat is resorbed and large fat volume cannot be transferred at each surgical procedure, several interventions are required. Therefore, adipose tissue engineering may provide a good alternative. Currently, new clinical solutions are under development using bioactive products that can generate adipose tissue. ${ }^{2}$ In some settings, poly(lactic-co-glycolic acid)
(PLGA) is used as a scaffold for seeding cells in vitro and then reimplanted for a longer term to engineer adipose tissue in vivo. ${ }^{3,4}$ To assess the amount and the quality of adipose tissue formed, we performed a histomorphometric analysis on all tissue sections. However, this experimental process is time consuming and not suitable for the examination of large volumes of fat. In addition, it leads to the sacrifice of many animals to obtain data from sequential measurements. In this study, we used PLGA as a vehicle to deliver fat component (oleic acid [OA]) in situ to the target tissue with the aim to evaluate the accuracy of a three-dimensional (3D) microcomputed tomography (CT) scan to follow in vivo biomaterials and fat volume changes. The advantages are the long-term follow-up of fat volume in the same animals, which allows

\footnotetext{
${ }^{1}$ Division of Plastic, Reconstructive and Aesthetic Surgery, Geneva University Hospitals and Medical School, University of Geneva, Geneva, Switzerland.

${ }^{2}$ Laboratoire des Polymères STI-IMX_LP, Ecole Polytechnique Fédérale de Lausanne, Institut des Matériaux and Institut des Sciences et Ingénierie Chimiques, Lausanne, Switzerland.

${ }^{3}$ Centre for BioMedical Imaging, Geneva University Hospitals, Geneva, Switzerland.
}

(C) Sarah Berndt et al. 2017; Published by Mary Ann Liebert, Inc. This is an Open Access article distributed under the terms of the Creative Commons Attribution License, which permits unrestricted use, distribution, and reproduction in any medium, provided the original work is properly cited.

*This article is part of a special focus issue on Animal Models in Tissue Engineering. Part II. 
both to analyze the scientific values of the measurements and to reduce the number of animals used in in vivo protocols according to the 3 " $\mathrm{R}$ " regulations governing animal ethics. To validate our technique, our PLGA was loaded with OA, as well as other different commercial soft tissue fillers, to assess the behavior of our new biomaterial to increase adipose tissue in situ by the injection of biodegradable microspheres loaded with lipids.

\section{Materials and Methods}

\section{Biodegradable microspheres loaded with lipids}

We developed a resorbable synthetic PLGA microsphere loaded with OA. When injected, these microspheres deliver free fatty acids to the adipocytes in situ, thus allowing local fat tissue augmentation. Blank PLGA microspheres were prepared by the single emulsion $(\mathrm{O} / \mathrm{W})$-solvent evaporation method as described elsewhere. ${ }^{5}$ The OA-loaded PLGA microspheres were prepared by mixing OA with the PLGAcontaining dichloromethane phase $(\mathrm{O})$. The size, surface aspect, and the content of the microspheres were characterized. The morphological examination of blank and OA-loaded microspheres was performed using a MERLIN (Carl Zeiss, Germany) scanning electron microscope as already described. ${ }^{6}$ Particle size is one of the most influential characteristics of the microspheres. Size distribution was measured by laser diffraction using Mastersizer S (Malvern Instruments Ltd., United Kingdom). To inject the smallest amount of microspheres with maximum efficacy, it is crucial to obtain the highest concentration of OA loaded on microspheres. Thus, the OA mass was increased from $1 \%, 2 \%$, and $3 \%$ to $7 \%$ to obtain microspheres with the highest loading efficiency (LE) and encapsulation efficiency (EE); quantitative characterization of the OA loading of the microspheres was obtained using nuclear magnetic resonance (NMR) spectroscopy.

\section{Animal experiments}

The concept of the innovation was to increase in situ fat tissue by the transfer of OA molecules from PLGA microspheres to the adipocytes located in the injection site. To assess the efficacy and safety of the OA-loaded microspheres, the volume of the inguinal fat pad of mice injected with OA was analyzed and compared to control groups. Fat volume changes and microsphere resorption were measured using a 3D micro-CT.
Animals. In this study, 72 12-week-old BALB C/J female wild-type mice (Janvier Labs, France) were used. They were kept under normal maintenance in a clean facility at the University of Geneva Animal Facility. All procedures regarding animal maintenance and experimental use were conducted following local regulations and ethics committee approval (GE49/15). Mice were anesthetized with $2 \%$ isoflurane during the surgical procedure and micro-CT assessment.

In vivo injection of microspheres containing OA compared to microsphere and control fillers. The behavior of microspheres containing OA was compared to an "injection solution" $(0.5 \% \mathrm{NaCl}$ with $1 \%$ Tween 80$)$ as a control, empty microspheres, hyaluronic acid (Juvederm ULTRA $4^{\circledR}$, Allergan, Ireland), and poly-L-lactic acid (PLA) (Sculptra ${ }^{\circledR}$; Sinclair Pharma, France). At present, the two latter products are considered to be the state-of-the-art fillers on the market. Juvederm ULTRA 4 is a highly cross-linked hyaluronic acid, which is resorbed after 3-6 months. Sculptra is a PLA, a biopolymer comparable to PLGA and resorbed after 6-12 months. However, it can offer long-term results due to collagen production provoked by inflammatory reactions.

To obtain a homogenous injectable product, the powder obtained following the technique described above was resuspended in the "injection solution" and filtered using $70 \mu \mathrm{m}$ Corning ${ }^{\circledR}$ cell strainer (Falcon) to obtain a final concentration of $135 \mathrm{mg}$ of $\mathrm{OA} / \mathrm{mL}$. According to the manufacturers' instructions for clinical use, Sculptra was suspended at a concentration of $16.6 \mathrm{mg} / \mathrm{mL}$, and the Juvederm ULTRA 4 was used without resuspension (Table 1). All products were injected through a $24 \mathrm{G}$ sharp needle connected to a $1 \mathrm{~mL}$ Luer Lock syringe and Lipopen ${ }^{\circledR}$ (Juva+, Switzerland). Lipopen is an innovative motorized cordless device that permits the homogenous injection of precise amount of a product contained in a syringe plugged into it. Apart from Juvederm ULTRA 4, the total volume of each injected product was $100 \mu \mathrm{L}$ per inguinal fat pad. As Juvederm ULTRA 4 was not diluted, the injected volume was $40 \mu \mathrm{L}$. To track the fat volume and polymer resorption over time, the $72 \mathrm{BALB} \mathrm{C} / \mathrm{J}$ mice (12 weeks old; weight: $18-23 \mathrm{~g}$ ) were randomly assigned to six different experimental conditions (12 animals per group), that is, naive, saline, empty microspheres, OA-loaded microspheres, Juvederm $^{\circledR}$, and Sculptra. The left inguinal fat pad (LIFP) was used as the "treatment" side and the right inguinal fat pad (RIFP) as an internal control where no injection was performed.

TABle 1. In ViVo EXPERIMENTAL Conditions

\begin{tabular}{|c|c|c|c|c|}
\hline $\begin{array}{l}\text { Experimental } \\
\text { conditions }\end{array}$ & $\begin{array}{l}\text { No. of } \\
\text { mice }\end{array}$ & $\begin{array}{l}\text { Nature of } \\
\text { product }\end{array}$ & $\begin{array}{l}\text { Quantity of product } \\
\text { injected in }(100 \mu L)\end{array}$ & $\begin{array}{l}\text { Injection } \\
\text { system }\end{array}$ \\
\hline Naïve & 12 & - & - & - \\
\hline Saline solution & 12 & $+0.1 \%$ Tween & $\overline{a x}$ & 24G needles \\
\hline Empty MS & 12 & $\begin{array}{l}\text { PLGA 1\% } \\
\text { Mannitol 0.5\% } \\
\text { CMC }\end{array}$ & $36.5 \mathrm{mg}$ MS & $24 \mathrm{G}$ needles \\
\hline Microspheres+OA & 12 & $\begin{array}{c}\text { PLGA+OA } \\
1 \% \text { mannitol } \\
0.5 \% \text { CMC }\end{array}$ & $50 \mathrm{mg}(13.5 \mathrm{mg} \mathrm{OA}+36.5 \mathrm{mg} \mathrm{MS})$ & 24G needles \\
\hline Sculptra & 12 & Polylactic acid & $1.66 \mathrm{mg}$ & 24G needles \\
\hline Juvederm & 12 & Hyaluronic acid & $40 \mu \mathrm{L}(6 \mathrm{mg})$ & 24G needles \\
\hline
\end{tabular}

CMC, carboxymethylcellulose; MS, microspheres; OA, oleic acid; PLGA, poly(lactic-co-glycolic acid). 
FIG. 1. Scanning electron microscopic images of blank (a) and oleic-acid-loaded (b) poly(lactic-co-glycolic acid) microspheres prepared by single emulsion $(\mathrm{O} / \mathrm{W})$ solvent evaporation method.
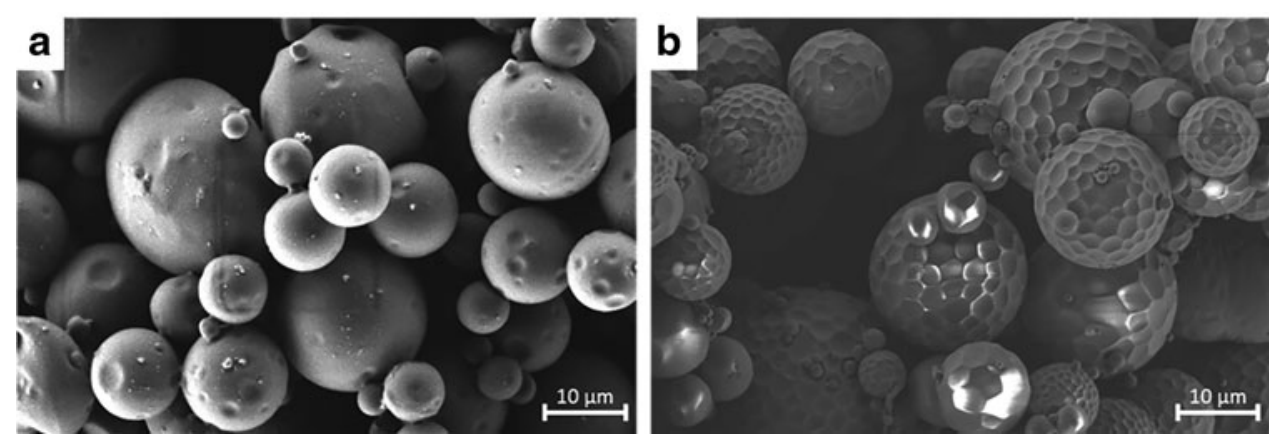

CT scan acquisition and quantification. Mice were anesthetized with $2 \%$ isoflurane and then subjected to CT scans in a Triumph micro-positron emission tomography (PET)/single photon emission computed tomography (SPECT)/CT system (TriFoil). Abdominal scans were obtained by placing the animal comfortably on its back in the animal holder and aligning its longitudinal axis parallel to the image plane. The upper limit area of scanning was set at the second lumbar vertebra using a two-dimensional (2D) prescan image. Scans were then obtained at 80 peak kilovoltage $(\mathrm{kVp})$ and $160 \mu \mathrm{A}$, and 1024 projections were acquired during a $360^{\circ}$ rotation with a field view of $71.3 \mathrm{~mm}$ (1.7 $\times$ magnification). CT scan analysis was performed at day 0 before injection and then at day 15, 30, 60, and 90 .

The Triumph XO software was used to reconstruct the CT scans with a matrix of 512 and a voxel size of $0.105 \mathrm{~mm}$. CT scans were then exported as Digital Imaging and Communications in Medicine (DICOM) files through the software Amira (FEI). The software OsiriX (Pixmeo) was used to quantitatively analyze the datasets and generate images. Regions of interest (ROI) were drawn on contiguous slices on CT-scans and computed as 3D volumes for the measurement of volumes and densities of the indicated adipose tissues. The contrast difference was used to discriminate the fat tissue versus the injected products (saline solution, empty microspheres, or OA-loaded microspheres).

ROI in the inguinal fat pad areas were delineated on 52 images ( 1 image of every 10 , evenly spaced across the whole animal depth) on 2D sections on both sides. The upper and lower limits were set at similar levels of depths between the animals. The algorithm used by the software then automatically reconstructed the $3 \mathrm{D}$ structure and gave precise quantification of the volume. CT recognizes different tissues upon their attenuation for X-rays in correlation to their densities, which are expressed in Hounsfield units (HU). In our study, muscle attenuation coefficients were set to 0 to allow direct comparison with fat and polymer attenuation coefficients.

\section{Statistical analysis}

One-way analysis of variance (ANOVA) was used for multiple comparisons in experiments with one independent variable. Results are expressed as mean \pm standard error of the mean (SEM). Dunnett's test was used for post hoc analysis of the significant ANOVA. A difference in mean values between groups was considered to be significant when $p<0.05$.

\section{Results}

\section{Microsphere properties and characterization}

The morphological examination demonstrated that blank PLGA microspheres were spherical and had a smooth surface without pores or cavities. The OA-loaded microspheres showed a spherical shape with a golf ball-like dimpled surface (Fig. 1). The size distribution of the blank and OAloaded microspheres presented a Gaussian distribution. The mean volume diameter was $39 \mu \mathrm{m}$ for blank microspheres and $41 \mu \mathrm{m}$ for OA-loaded microspheres with a span value of 4.2 and 1 , respectively.

The LE increased linearly for the formulation of 1-3\% and formed a plateau with 7\% OA loading (Fig. 2). The handling of particles obtained with a $7 \%$ formulation was difficult, and a change in the morphology of the microspheres was visible macroscopically. The change in particle characteristics of microspheres obtained with the $7 \%$ formulation was also confirmed by laser diffraction analysis. As shown in Table 2, when the drug mass was raised from $1 \%$ to $3 \%$, the LE increased at the same rate with a quantitative EE. At a certain ratio of OA to PLGA, the LE reaches a plateau. When further increasing the drug mass to $6.7 \%$, the PLGA microspheres could no longer encapsulate the OA acid efficiently and the EE decreased to almost $50 \%$. Based on these results, a $3 \%$ formulation with $75-80 \%$ LE and $100 \%$ EE was chosen for all further experiments.

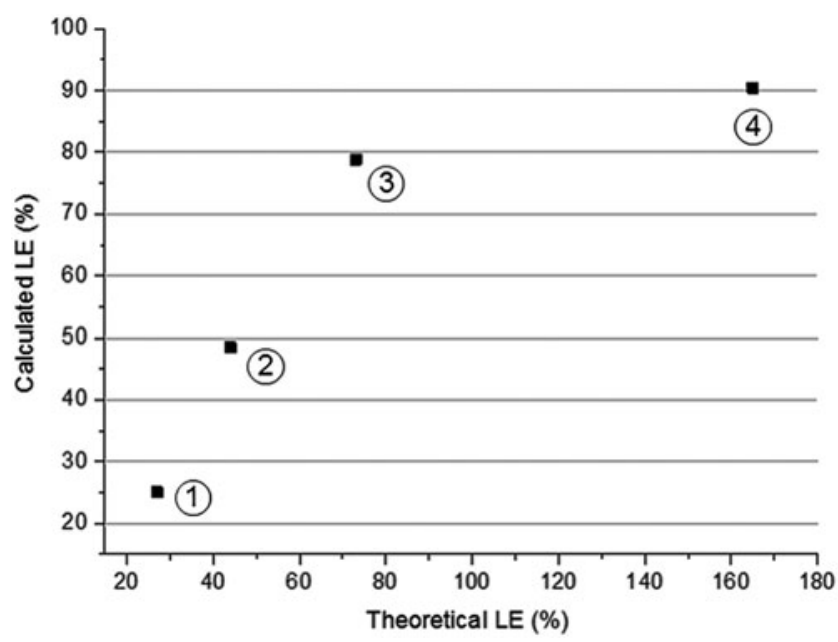

FIG. 2. Graphical representation of the obtained LE versus theoretical LE using formulations 1-4. LE, loading efficiency. 
Table 2. Formulations of Oleic Acid-Loaded Poly(Lactic-Co-Glycolic Acid) Microspheres

\begin{tabular}{lcccccr}
\hline Formulation & $\begin{array}{c}\text { PLGA } \\
\text { concentration }(w / v)\end{array}$ & $\begin{array}{c}\text { OA } \\
\text { concentration }(w / v)\end{array}$ & $\begin{array}{c}\text { Mean volume } \\
\text { diameter }(\mu m)\end{array}$ & $\begin{array}{c}\text { Span } \\
\text { value }\end{array}$ & LE (\%) & EE (\%) \\
\hline 1 & $4 \%$ & $1.1 \%$ & 39.1 & 1.4 & 25 & 94 \\
2 & $4 \%$ & $1.8 \%$ & 45.3 & 1.3 & 48 & 110 \\
3 & $4 \%$ & $3.0 \%$ & 57.0 & 1.4 & 79 & 109 \\
4 & $4 \%$ & $6.7 \%$ & 177.3 & 3.4 & 90 & 55 \\
\hline
\end{tabular}

EE, encapsulation efficiency; LE, loading efficiency.

Animal experiments: in vivo micro-CT scan assessment and ex vivo histological analysis

The subcutaneous inguinal fat pad and the injected material were easily distinguishable from the surrounding tissues by different tissue density images detected on the CT scan (Fig. 3). Fat tissues ranged between -125 and $-150 \mathrm{HU}$ in our measurements, while polymers ranged between -25 and $-39 \mathrm{HU}$. This attenuation coefficient differences allowed to precisely depict small amounts of polymer entrapped within the fat. In Figure 4, we show how we delineated the ROI on the control RIFP and on the LIFP to be injected on the 512 2D images (Fig. 4a) and how the software directly reconstructed the entire fat pad in 3D (Fig. 4b). After empty microsphere or OA-loaded microsphere injection, the microCT scan allowed to detect the presence of the product within the fat tissue. As shown in Figure 5, PLGA has a radiopacity quite similar to muscle, while fat is a soft tissue and presents lower values of HU (Fig. 5).

Inguinal fat pad quantification. The noninjected contralateral control RIFP showed a tendency to increase over time, which could be attributed to normal weight gain increase (less than $10 \%$ increase between the different time points in all the experimental groups) (Fig. 6).

Injected fat pads showed increased volumes in all groups. Naive fat pads and those with the "injection solution" as the control and Sculptra had the same tendency as the RIFP, that is, a continuous fat pad volume increase of up to $10 \%$ at day 90. Fat pads injected with the empty microspheres peaked at day 15 with a $76 \%$ volume increase and then showed a

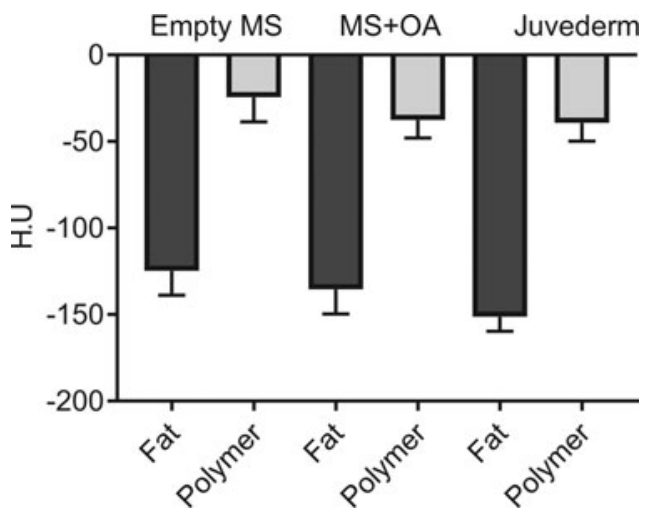

FIG. 3. Micro-CT scan quantification of the densities of the different tissues of interest in HU. Calculations were normalized to muscle attenuation coefficients that were set to 0 . CT, computed tomography; HU, Hounsfield units. reduction at day 90 with $68 \%$ of fat pad increase. In the OAloaded microsphere group, the volume increase peaked at day $30(87 \%)$ and then reduced to $45 \%$ at day 90 . In the Juvederm group, the volume increased at day 15 and remained stable until day 90 with an $81 \%$ volume increase (Fig. 7).

Injected product resorption results. To assess the product resorption, the volume of the injected material over time was plotted (Fig. 8). The synthetic material was only detectable in the empty microsphere, OA-loaded microsphere, and Juvederm ULTRA 4 groups. Resorption was almost complete after 3 months for the empty microsphere and OA-loaded microsphere groups. In the empty microsphere group, we observed $18 \%$ volume resorption after 30 days, $64 \%$ after 60 days, and $75 \%$ after 90 days. In the OA-loaded microsphere group, the resorption rate was $25 \%$ after 30 days, $70 \%$ after 60 days, and $93 \%$ after 90 days.

In comparison, the highly cross-linked hyaluronic acid (Juvederm) remained more stable over time: 10\% resorption after 30 days, $54 \%$ after 60 days, and 56\% after 90 days. Sculptra did not show any obvious polymer residue in the fat pad.

\section{Discussion}

In situ adipose tissue engineering is of prime importance because of the increasing need for soft tissue filler procedures. $^{7}$ Currently, most adipose tissue engineering approaches imply the use of living cells and/or biomolecules and/or biocompatible scaffolds. ${ }^{8}$ Before any clinical use of these approaches, the assessment of these components after in vivo implantation is mandatory. To our knowledge, little has been reported on the in vivo follow-up of these techniques in animal models.

Three-dimensional micro-CT has already been used by Phipps et al. to follow the evolution of fat graft implanted without any biomaterial under the scalp of mice. ${ }^{9}$ Previous studies have also shown high correlations between microCT-derived fat pad volumes and their weight after excision,${ }^{10-13}$ thus highlighting the accuracy of this noninvasive technique. Micro-CT scan density analyses can also be used to monitor the activity and browning of white fat pads, which have been correlated to their densities. ${ }^{14-16}$

Our technique is novel in terms of in vivo tracking of both biological and synthetic components of engineered tissue over a long period of time. The 3D micro-CT scan method showed a high accuracy and allowed to follow polymer degradation in vivo, as well as fat volume changes, while animals required only a mild anesthesia, and there was no need for a contrast agent. 
FIG. 4. Micro-CT scan images of the abdominal mouse area of a naive mouse (no biomaterial injected). ROI are drawn sequentially on in-depth 2D pictures on one picture every 10 , out of a total of 512 coronal slices (a). OsiriX software automatically reconstructs the $3 \mathrm{D}$ pictures and hence allows precise volume quantification (b). 2D, two-dimensional; 3D, three-dimensional; LIFP, left inguinal fat pad; RIFP, right inguinal fat pad; ROI, regions of interest.
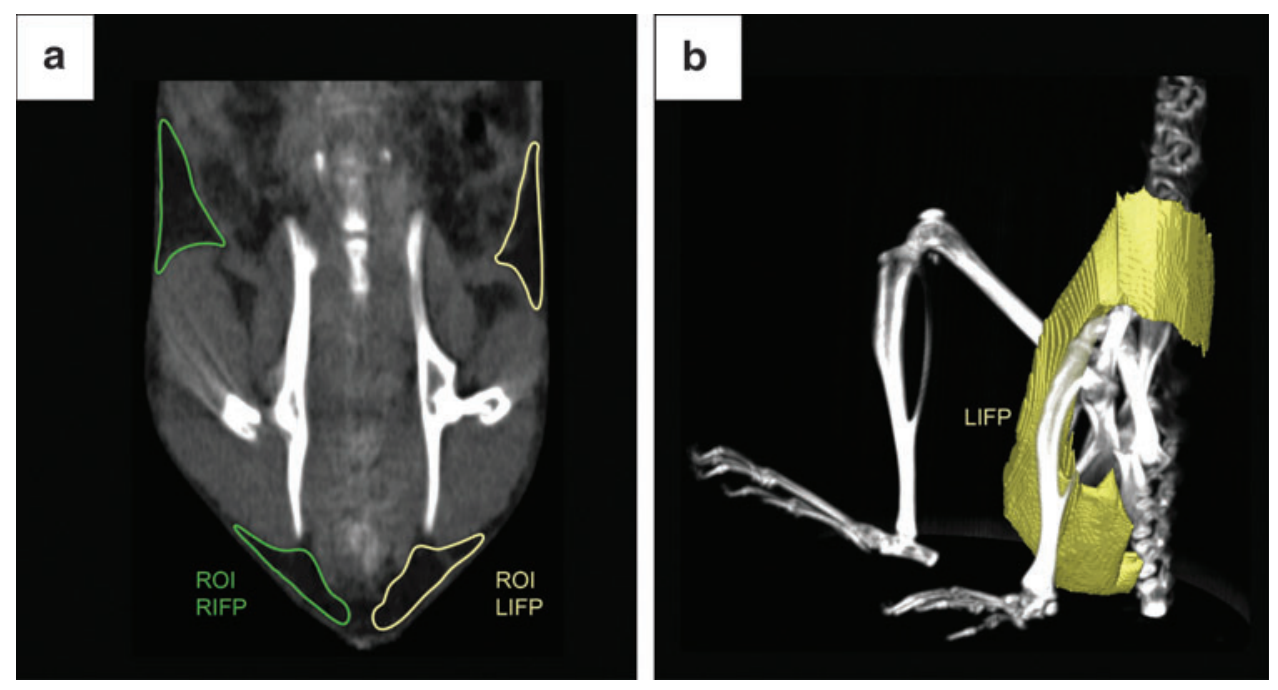

The degradation rate of PLGA depends on the copolymer composition and microsphere size. PLGA is a drug release system of choice, which is degraded by the hydrolysis of the ester bonds into lactic and glycolic acids and, ultimately, metabolized to carbon dioxide and water. ${ }^{17}$ The copolymer
PLGA has been already used for engineered constructs, featuring human-rat adipocytes and an additional angiogenic growth factor (basic fibroblast growth factor [bFGF]) on a 3D scaffold to induce neovascularization in vivo. ${ }^{18,19}$ In another study, PLGA seeded with mesenchymal stem cells
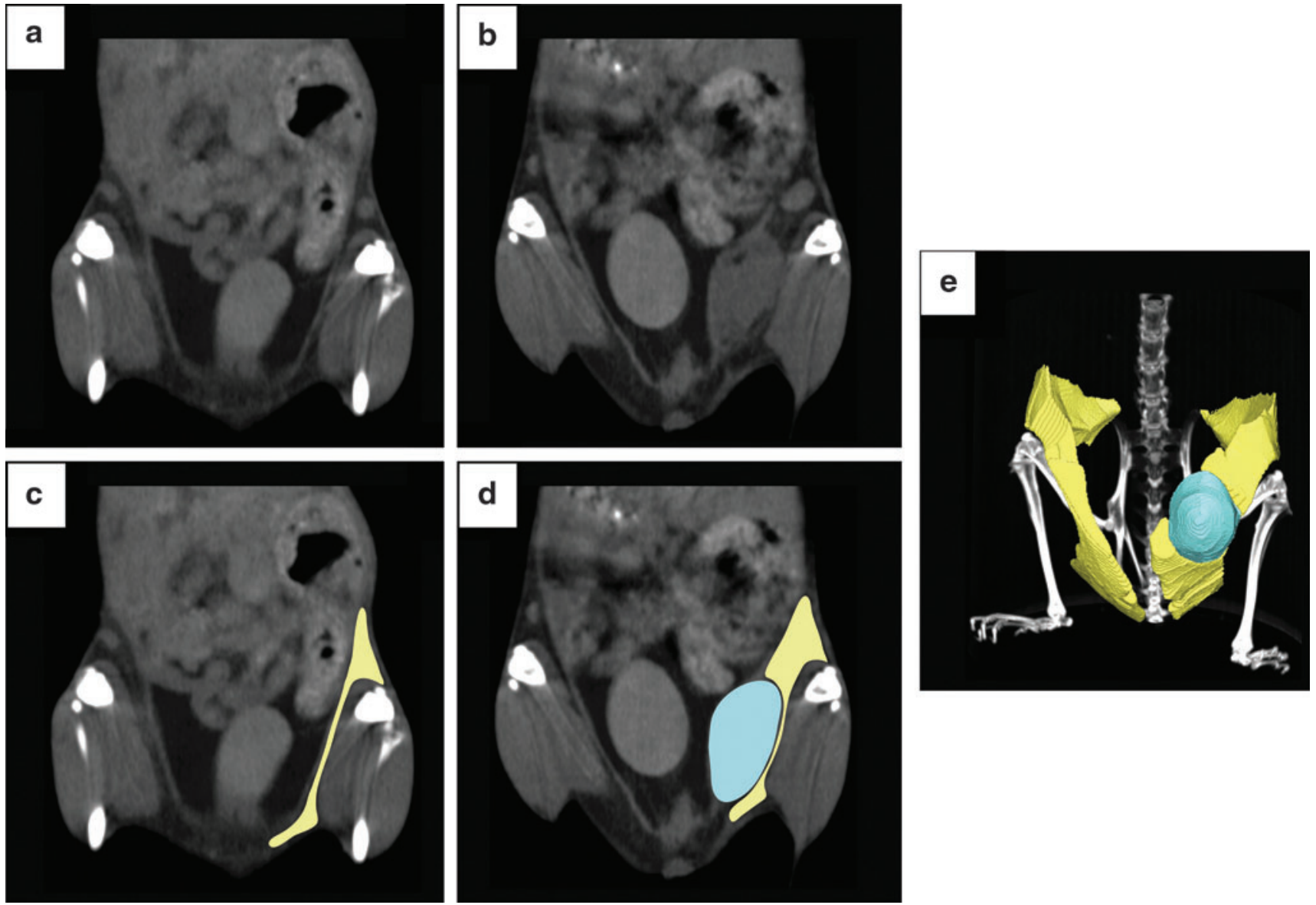

FIG. 5. Micro-CT scan image analysis of the abdominal mouse area of an empty microsphere-injected mouse before injection at day 0 (a), after 15 days of injection (b). Before injection, only fat tissue is evidenced in the inguinal area in green (c). At day 15, a clear contrast difference of the fat (green) and the injected material (blue) allows a precise delineation of the ROI (d). Three-dimensional reconstruction of the control side noninjected (RIFP) and the injected site (LIFP) (e). 


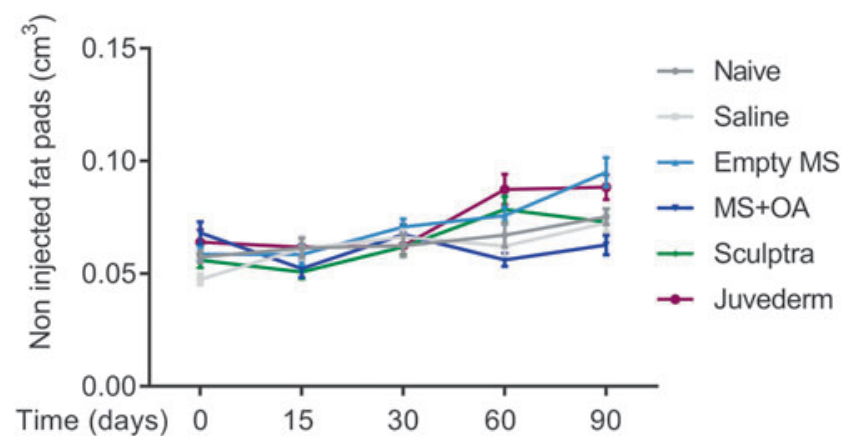

FIG. 6. Noninjected fat pad quantification in the different experimental conditions along time. $n=12$ animals per group. Error bars =SEM. SEM, standard error of the mean.

was injected and newly formed tissue was assessed only once postmortem. $^{20}$ Recently, a group injected dibenzazepineloaded PLGA microspheres in the same target tissue to assess fat browning in situ. They did not follow the polymer in vivo and rather used fluorescent microscopy after the sacrifice. ${ }^{21}$

In our formulation, we designed microspheres that have to be degraded over 3 months, which would allow a sustained in vivo OA delivery of up to 3 months. Therefore, a 50:50 lactic to glycolide ratio was used to produce microspheres with a homogenous size of $30-50 \mu \mathrm{m}$. According to the literature, this copolymer ratio and size are known to offer microspheres that are degraded over 2-3 months. ${ }^{22,23}$ In our study, the 3D micro-CT scan allowed to confirm that these microspheres are resorbed over 90 days. By contrast, high cross-linked HA, which is known to be degraded over a longer time, was not resorbed completely at 90 days. The CT images allow an accurate distinction of the various body compartments at the tissue/organ level, including adipose tissue. As quantitative measurements present a high accuracy and reproducibility, a CT scan is now recognized as the gold standard in medical imaging. ${ }^{24,25}$

The aim of this study was to assess quantitatively the efficacy of the micro-CT scan technique to track PLGA polymer in vivo and to follow its resorption, as well as to be able to procure further evidence of fat pad volume enhancement. The main advantage of this technology is the in vivo tracking

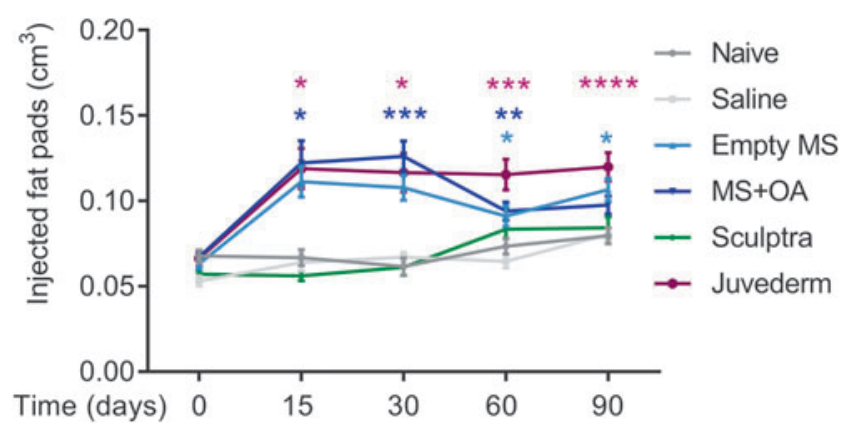

FIG. 7. Injected fat pad quantification in the different experimental conditions along time. $n=12$ animals per group. Error bars = SEM. One-way ANOVA followed by Dunnett's multiple comparison test against saline experimental condition. ${ }^{*} p<0.05, * * p<0.01$, $* * * p<0.001$, and $* * * * p \leq 0.0001$. ANOVA, analysis of variance.

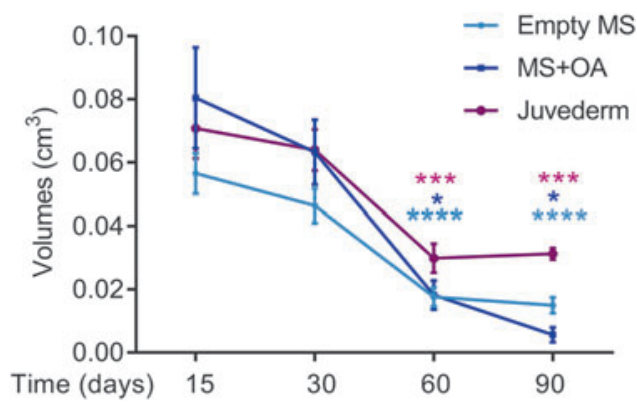

FIG. 8. Product resorption results. $n=12$ animals per group. Error bars = SEM. One-way ANOVA followed by Dunnett's multiple comparison test. In each experimental group, comparison on the different time points against day 15 . $* p<0.05$, $* * p<0.01, * * * p<0.001$, and $* * * * p \leq 0.0001$.

of both the injected polymer and the fat target tissue. It offers the possibility to follow the same animal over a long period of time. In addition, this long-term noninvasive monitoring required the enrollment of fewer animals in the in vivo protocol because it was not necessary to sacrifice animals to obtain measurements. The sensitivity of the technique was proved by the observation of subtle fat pad changes (smallest difference observed, $0.98 \mathrm{~mm}^{3}$ ), including the smallest volume of synthetic volumes $\left(1.26 \mathrm{~mm}^{3}\right)$ detected and followed for resorption. Nevertheless, the main disadvantage of this technique is the difficulty to assess in vivo the quality of fat (i.e., apoptosis, angiogenesis), which requires histological analysis.

To stick to the clinical protocol for Sculptra use, the concentration of PLA was low. This low polymer concentration of PLA in Sculptra (e.g., 20 times less than microsphere or OA-loaded microsphere formulations) could not be detected by $3 \mathrm{D}$ CT scan and it is probable that this volume of synthetic material $\left(<0.5 \mathrm{~mm}^{3}\right)$ is the inferior limit of detection by this technique. However, the highly cross-linked HA (Juvederm) could easily be tracked in vivo over time. We observed a stable volume up to 30 days with resorption starting from that time point. The behavior of this product corresponds to our clinical experiment and the company documentation, that is, resorption of the product after 4-6 months.

\section{Conclusion}

Our in vivo model of fat pad injection was coupled with a long-term in vivo tracking of the biomaterial using a microCT scan approach. Automatic 3D reconstruction allowed a precise assessment of the fat and biopolymer volumes. We consider that this methodology represents a good platform to track in vivo biodegradable products and to assess the efficacy of adipose tissue engineering products for a bench-tobed approach.

\section{Acknowledgment}

The project was supported by the Technology and Information Commission, Switzerland (CTI no. 17520.1).

\section{Disclosure Statement}

No competing financial interests exist. 


\section{References}

1. Pu, L.L., Yoshimura, K., and Coleman, S.R. Fat grafting: current concept, clinical application, and regenerative potential, part 1. Clin Plast Surg 42, ix, 2015.

2. Choi, J.H., Gimble, J.M., Lee, K., Marra, K.G., Rubin, J.P., Yoo, J.J., Vunjak-Novakovic, G., and Kaplan, D.L. Adipose tissue engineering for soft tissue regeneration. Tissue Eng Part B Rev 16, 413, 2010.

3. Choi, Y.S., Park, S.N., and Suh, H. Adipose tissue engineering using mesenchymal stem cells attached to injectable PLGA spheres. Biomaterials 26, 5855, 2005.

4. Patrick, C.W., Jr., Zheng, B., Johnston, C., and Reece, G.P. Long-term implantation of preadipocyte-seeded PLGA scaffolds. Tissue Eng 8, 283, 2002.

5. Wang, Y., Gu, B., and Burgess, D.J. Microspheres prepared with PLGA blends for delivery of dexamethasone for implantable medical devices. Pharm Res 31, 373, 2014.

6. Ravi, S., Peh, K.K., Darwis, Y., Murthy, B.K., Singh, T.R., and Mallikarjun, C. Development and characterization of polymeric microspheres for controlled release protein loaded drug delivery system. Indian J Pharm Sci 70, 303, 2008.

7. Bajek, A., Gurtowska, N., Olkowska, J., Kazmierski, L., Maj, M., and Drewa, T. Adipose-derived stem cells as a tool in cell-based therapies. Arch Immunol Ther Exp (Warsz) 64, 443, 2016.

8. Casadei, A., Epis, R., Ferroni, L., Tocco, I., Gardin, C., Bressan, E., Sivolella, S., Vindigni, V., Pinton, P., Mucci, G., and Zavan, B. Adipose tissue regeneration: a state of the art. J Biomed Biotechnol 2012, 462543, 2012.

9. Phipps, K.D., Gebremeskel, S., Gillis, J., Hong, P., Johnston, B., and Bezuhly, M. Alternatively activated M2 macrophages improve autologous fat graft survival in a mouse model through induction of angiogenesis. Plast Reconstr Surg 135, 140, 2015.

10. Chung, M.T., Hyun, J.S., Lo, D.D., Montoro, D.T., Hasegawa, M., Levi, B., Januszyk, M., Longaker, M.T., and Wan, D.C. Micro-computed tomography evaluation of human fat grafts in nude mice. Tissue Eng Part C Methods 19, 227, 2013.

11. Judex, S., Luu, Y.K., Ozcivici, E., Adler, B., Lublinsky, S., and Rubin, C.T. Quantification of adiposity in small rodents using micro-CT. Methods 50, 14, 2010.

12. Lu, L., Peter, S.J., Lyman, M.D., Lai, H.L., Leite, S.M., Tamada, J.A., Uyama, S., Vacanti, J.P., Langer, R., and Mikos, A.G. In vitro and in vivo degradation of porous poly (DL-lactic-co-glycolic acid) foams. Biomaterials 21, 1837, 2000.

13. Wyatt, S.K., Barck, K.H., Kates, L., Zavala-Solorio, J., Ross, J., Kolumam, G., Sonoda, J., and Carano, R.A. Fullyautomated, high-throughput micro-computed tomography analysis of body composition enables therapeutic efficacy monitoring in preclinical models. Int $\mathrm{J}$ Obes (Lond) 39, 1630, 2015.

14. Baba, S., Jacene, H.A., Engles, J.M., Honda, H., and Wahl, R.L. CT Hounsfield units of brown adipose tissue increase with activation: preclinical and clinical studies. J Nuclear Med 51, 246, 2010.

15. Chevalier, C., Stojanovic, O., Colin, D.J., Suarez-Zamorano, N., Tarallo, V., Veyrat-Durebex, C., Rigo, D., Fabbiano, S.,
Stevanovic, A., Hagemann, S., Montet, X., Seimbille, Y., Zamboni, N., Hapfelmeier, S., and Trajkovski, M. Gut microbiota orchestrates energy homeostasis during cold. Cell 163, 1360, 2015.

16. Suarez-Zamorano, N., Fabbiano, S., Chevalier, C., Stojanovic, O., Colin, D.J., Stevanovic, A., Veyrat-Durebex, C., Tarallo, V., Rigo, D., Germain, S., Ilievska, M., Montet, X., Seimbille, Y., Hapfelmeier, S., and Trajkovski, M. Microbiota depletion promotes browning of white adipose tissue and reduces obesity. Nat Med 21, 1497, 2015.

17. Makadia, H.K., and Siegel, S.J. Poly lactic-co-glycolic acid (PLGA) as biodegradable controlled drug delivery carrier. Polymers 3, 1377, 2011.

18. Cho, S.W., Song, K.W., Rhie, J.W., Park, M.H., Choi, C.Y., and Kim, B.S. Engineered adipose tissue formation enhanced by basic fibroblast growth factor and a mechanically stable environment. Cell Transplant 16, 421, 2007.

19. Neubauer, M., Hacker, M., Bauer-Kreisel, P., Weiser, B., Fischbach, C., Schulz, M.B., Goepferich, A., and Blunk, T. Adipose tissue engineering based on mesenchymal stem cells and basic fibroblast growth factor in vitro. Tissue Eng 11, 1840, 2005.

20. Choi, Y.S., Park, S.N., and Suh, H. The effect of PLGA sphere diameter on rabbit mesenchymal stem cells in adipose tissue engineering. J Mater Sci Mater Med 19, 2165 , 2008.

21. Jiang, C., Kuang, L., Merkel, M.P., Yue, F., Cano-Vega, M.A., Narayanan, N., Kuang, S., and Deng, M. Biodegradable polymeric microsphere-based drug delivery for inductive browning of fat. Front Endocrinol 6, 169, 2015.

22. Middleton, J.C., and Tipton, A.J. Synthetic biodegradable polymers as orthopedic devices. Biomaterials 21, 2335, 2000.

23. Spenlehauer, G., Vert, M., Benoit, J.P., and Boddaert, A. In vitro and in vivo degradation of poly (D,L lactide/glycolide) type microspheres made by solvent evaporation method. Biomaterials 10, 557, 1989.

24. Mazonakis, M., and Damilakis, J. Computed tomography: what and how does it measure? Eur J Radiol 85, 1499, 2016.

25. Shearer, T., Bradley, R.S., Hidalgo-Bastida, L.A., Sherratt, M.J., and Cartmell, S.H. Three-dimensional visualisation of soft biological structures by X-ray computed microtomography. J Cell Sci 129, 2483, 2016.

Address correspondence to: Ali Modarressi, MD, PD

Division of Plastic, Reconstructive and Aesthetic Surgery Geneva University Hospitals 4 Rue Gabriel-Perret-Gentil Genève 1211 Switzerland

E-mail: ali.modarressi@hcuge.ch

Received: April 8, 2017 Accepted: August 9, 2017 Online Publication Date: October 3, 2017 